\title{
Polymer nanofiber-carbon nanotube network generating circuits
}

\author{
Mustafa Umut MUTLU ${ }^{1, \text { a }}$, Osman AKIN ${ }^{2,5, b}$ and Ümit Hakan YILDIZ ${ }^{3,4,5, c}$ \\ ${ }^{1}$ Materials Science and Engineering, Izmir Institute of Technology, Urla, Izmir, Turkey \\ ${ }^{2}$ Department of Mechatronics, Katip Çelebi University, Urla, Izmir, Turkey \\ ${ }^{3}$ Department of Chemistry, Izmir Institute of Technology, Urla, Izmir, Turkey \\ ${ }^{4}$ Department of Photonics, Izmir Institute of Technology, Urla, Izmir, Turkey \\ ${ }^{5}$ Inovasens Bilgi Tek. LTD. Izmir Technopark, Urla, Izmir, Turkey \\ amustafamutlu@iyte.edu.tr, ${ }^{b}$ osman.akin@ikc.edu.tr, ${ }^{c}$ hakanyildiz@iyte.edu.tr
}

\begin{abstract}
The polymer nanofiber carbon nanotube (CNT) based devices attracts attention since they promise high performance for next generation devices such as wearable electronics, ultra-light weighted appliances and foldable devices. This abstract describes the utilization of polymer nanofibers and CNT as major component of low cost foldable photo-resistor. We use polymer nanofiber as template guiding CNTs to generate nanocircuits and conductive sensing network. The controlled combination of CNTs and polymer nanofibers provide opportunities for device miniaturization without loss of performance. The nanofiber-CNT network based photo-resistor exhibits broad band response 400 to $1600 \mathrm{~nm}$ that holding promises for ultra-thin devices and new sensing platforms.
\end{abstract}

Keywords: Polymeric Solar Cells, Electrospunned nanofibers, Semiconductors, Carbon Nanotube.

\section{INTRODUCTION}

Soft electronics emerging as a key technology that recover the mismatch between human body and rigid circuits/hard electronics. The growing demand to the wearable electronics recently drives the advancements in soft electronics which speeds up replacement of silicon based conventional technology. The mobile healthcare/continuous monitoring applications such as monitoring of motion, respiration, body temperature, as well as electrophysiological outputs are requiring miniaturized wearable sensors/actuators are leading the growing demand ${ }^{[1-5]}$. The role of the carbon nanomaterials -is getting pivotal since they exhibit high performance and desirable mechanical properties and therefore they usually utilized as major component in wearable sensors/actuators, memories, energy devices, and displays. The mobility of the carbon nanotubes (CNTs) provides excellent conductivity/very low resistivity while thickness is still incomparably thin than the bulk silicon. The CNTs on flexible substrates (e.g., polydimethylsiloxane, polyurethane and other elastomeric materials) are recently utilized as mechanical sensors, wearable displays and energy devices ${ }^{[6-10]}$.

In this paper we introduces an alternative approach for CNTs based flexible sensors that utilizes functional polymeric nanofiber of poly (vinyledenefluoride) PVDF as deformable substrate. The nanofibers exhibit high mechanical strength while providing desired flexibility as well as network structure leading interconnected CNT moieties in large area ${ }^{[11]}$. This unique network structure facilitate utilization of $2 \times 2 \mathrm{~cm}$ sized flexible conductive substrate without tedious fabrication steps.

We described the utilization of polymer nanofibers and CNT as major component of low cost foldable photo-resistor. We use polymer nanofiber as template guiding CNTs to generate nanocircuits and conductive sensing network. The controlled combination of CNTs and polymer nanofibers provide opportunities for device miniaturization without loss of performance ${ }^{[12-15]}$. The nanofiber-CNT network based photo-resistor exhibits broad band response 400 to $1600 \mathrm{~nm}$ that holding promises for ultra-thin wearable devices.

Organic Photonic Materials and Devices XX, edited by Christopher E. Tabor, François Kajzar,

Toshikuni Kaino, Yasuhiro Koike, Proc. of SPIE Vol. 10529, 105290R - (C) 2018 SPIE

CCC code: $0277-786 \mathrm{X} / 18 / \$ 18 \cdot$ doi: $10.1117 / 12.2289085$ 


\section{EXPERIMENT}

Poly vinyldifloridine (PVDF) (Mw = $275000 \mathrm{~g} / \mathrm{mol}$ ), CNT obtained from Sigma-Aldrich and used as it is. Silver paste, cupper tape and Poly Methylmetacrylate (PMMA) (3mm thickness) was purchased from local market.

\subsection{Electrospinning PVDF Nanofibers}

PVDF solution was prepared by dissolving $4.59 \mathrm{~g}$ PVDF in $12 \mathrm{~mL}$ DMF and $3 \mathrm{~mL}$ acetone and kept under stirring for 15 hours at $50{ }^{\circ} \mathrm{C}$ to obtain homogeneous solution. Then, it was loaded into $20 \mathrm{~mL}$ plastic syringe. Loaded syringe was connected to electrospinning instrument (Inovenso) with rotating collector. The voltage for electrospinning was $24 \mathrm{kV}$. The distance between the nozzle tip and the collector was $170 \mathrm{~mm}$. The rotating speed of collector was $500 \mathrm{rpm}$ to collect aligned PVDF nanofibers.

\subsection{Acid treatment of CNT}

To obtain well-dispersed CNT in aqueous solution, pristine CNTs were functionalized by chemical oxidation using nitric acid and sulfuric acid. The procedure of Yücel et all was applied for the treatment ${ }^{[16]}$.

\subsection{Fabricating PMMA Surface}

$3 \mathrm{~mm}$ PMMA plate was cut into $10.8 \times 7.2 \mathrm{~mm}$ outer frame. Center of the PMMA surface was engraved in $6.8 \times 3.2 \mathrm{~mm}$. The depth of engraved area was $1 \mathrm{~mm}$.

\subsection{Preparation of CNT based photo-resistor}

PMMA surface, functionalized with cupper tapes on both edges, was placed on the collector of the electrospinning instrument. Then, the same procedure on 2.1. was applied to obtain PVDF nanofibers hanging between edges of the PMMA surface. Each edge of the collected surface was painted with silver paste to make ohmic contact. After that, $40 \mu \mathrm{L}$ acid treated CNT dispersion in various concentrations were drop casted on the PVDF nanofibers. Finally, 4 hours of incubation at $60^{\circ} \mathrm{C}$ was performed to get conductive network.

\subsection{Measurements}

All the measurements have been performed instantaneously with Keysight Technologies 34401A Digital Multimeter. External DC supply was used for LEDs illumination. $405 \mathrm{~nm}, 465 \mathrm{~nm}, 525 \mathrm{~nm}$ and $625 \mathrm{~nm}$ light sources were bought from local market and $850 \mathrm{~nm}, 1200 \mathrm{~nm}, 1300 \mathrm{~nm}, 1450 \mathrm{~nm}, 1550 \mathrm{~nm}$ and $1600 \mathrm{~nm}$ IR light sources were supplied from Thorlabs.

\section{RESULTS}

As shown in figure 1 device structure is consist of CNT photo-resistor layer on top of electrospun PVDF nanofiber on PMMA substrate (PMMA used only to fix nanofiber during fabrication). Copper tapes on each edge provides the electric force that draw charged PVDF nanofibers during electrospinning process. On the other hand, the cavity in the inner frame of the PMMA substrate causes discontinuity in the force by creating a nonconductive air gap, and therefore, the combination causes an alignment of PVDF nanofibers between the conductive poles apart from inside the conductive poles as shown in figure $1(\mathrm{~b}$ and $\mathrm{c}$ ). Semi-aligned PVDF nanofibers form highly hydrophobic surface to hold drop casted aqueous CNT solution applied on and act as a substrate after volatilization of solvent in CNT solution. As shown in figure 1(d), solid CNTs tangled each other and PVDF nanofibers while volatilization and form a highly conductive network that sensitive to electromagnetic radiation. 


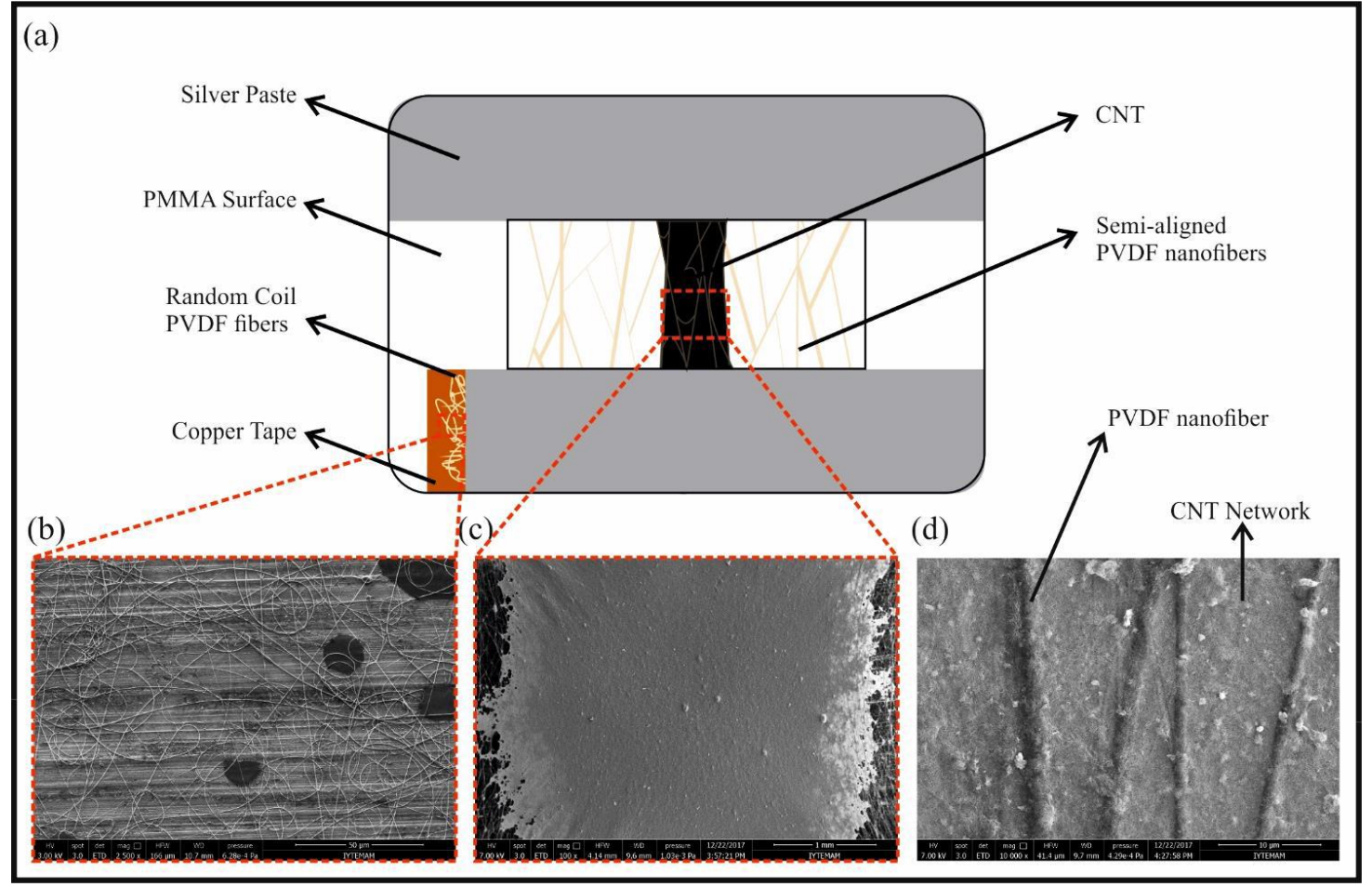

Figure1. Schematic illustration of photo-resistor structure (a). SEM images of random coil PVDF nanofibers on conductive copper surface after electrospinning process and CNT network on semi-aligned PVDF nanofibers (c and d).

To investigate the effect of the CNT concentration effect, photo-resistor devices were fabricated by using varying initial concentration of CNT stock solutions. Figure 2(a) illustrates the variation of resistivity with respect to the CNT concentration. The base resistivities were $9.62 \pm 0.48,3.02 \pm 0.15,1,08 \pm 0.05,0.50 \pm 0.02$ and $0.22 \pm 0.01$ for the CNT concentrations; $0.01 \%, 0.02 \%, 0.05 \%, 0.1 \%$ and $0.2 \%$, respectively. Lower CNT concentrations disrupt the homogeneity of the CNT network that causes disorder in base resistivity. On the other hand, higher CNT concentration offers more stable structure that resemble each other. Figure 2(b) plots the typical J-V curve of the Ag-CNT-Ag sandwich type ohmic interaction from $0 \mathrm{~V}$ to $4 \mathrm{~V}$ in different CNT concentrations in dark current. Photo-resistors show symmetric behavior under reverse bias as expected due to the lack of additional junction(s). 
(a)

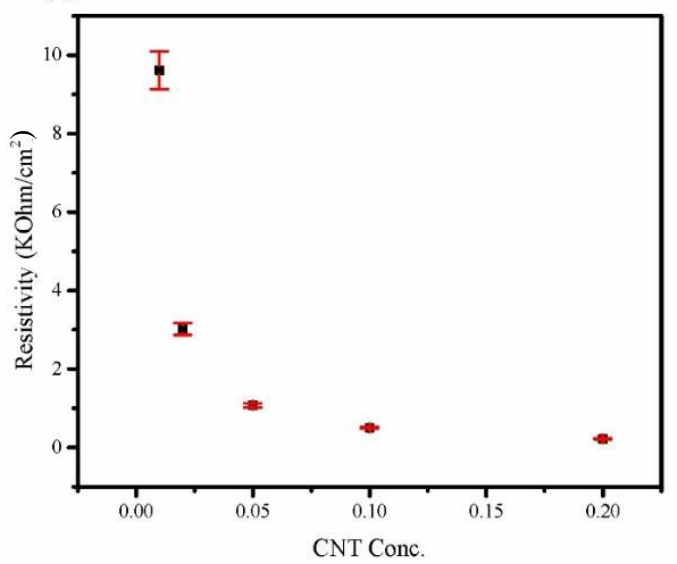

(b)

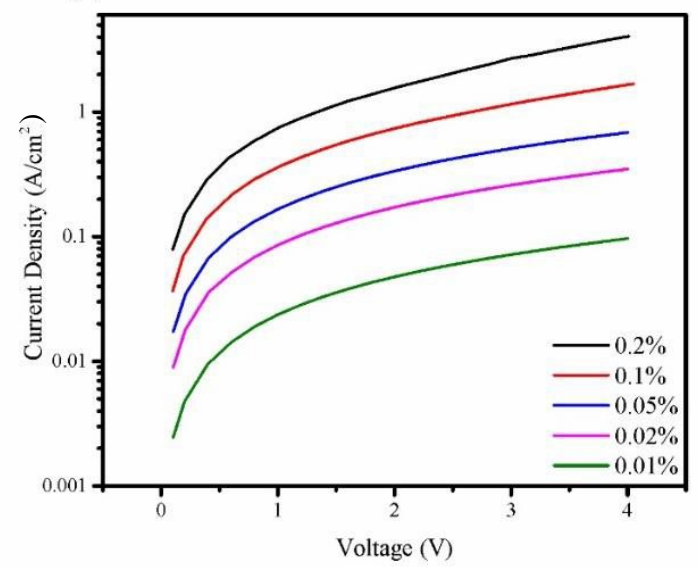

Figure 2. The influence of the CNT concentration on the base resistivity of the CNT photo-resistor (a). Typical room temperature J-V curves of the photo-resistors in dark current.

Characteristics of broadband photoresponse of photo-resistors as a function of incident wavelength is investigated by using LEDs from visible to infrared range (405, 465, 525, 625, 850, 1200, 1300, 1450, 1550 and 1600nm). Figure 3(a and b) plots the responsivities for various wavelengths which can be observed clearly that photo-resistors have a broadband sensitivity in the range from 400-1600 nm. Photovoltaic effect observed in the whole spectrum by applying a $1 \mathrm{~V}$ bias since the increasement of bias voltage causes in fluctuations even amplify the obtained photocurrent significantly (S1). As observed in figure $3 \mathrm{~b}$, photo-resistors have linearity in the infrared region on the contrary of the increasement in the wavelength of incident light. Surprisingly, 0.1\% CNT contained photo-resistor have higher responsivity than the one contains $0.2 \%$ CNT almost all incident wavelengths, which was showed particularly in figure $3 \mathrm{c}$ that illustrates the photocurrents of the photo-resisters in various CNT concentration illuminated by $850 \mathrm{~nm}$ light source with intensity of $25.9 \mathrm{~mW} / \mathrm{cm}^{2}$. The reason of this phenomenon is still unknown and needs further investigation. 
(a)

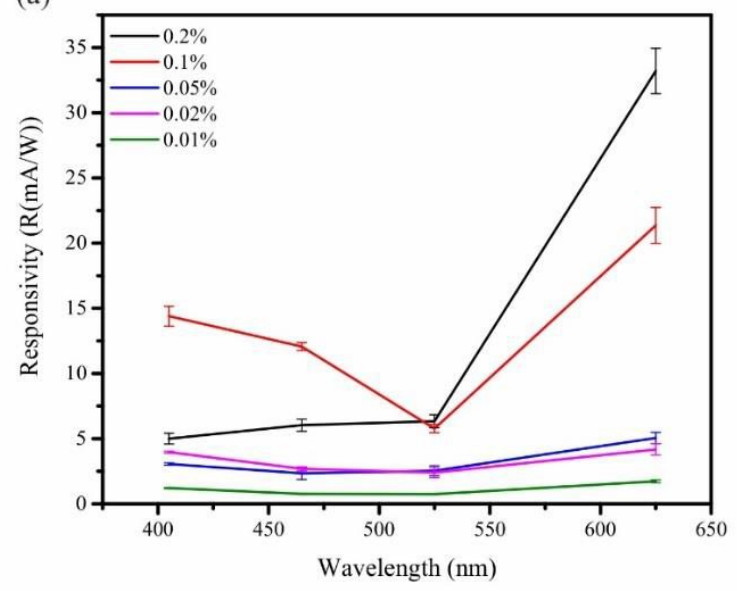

(b)

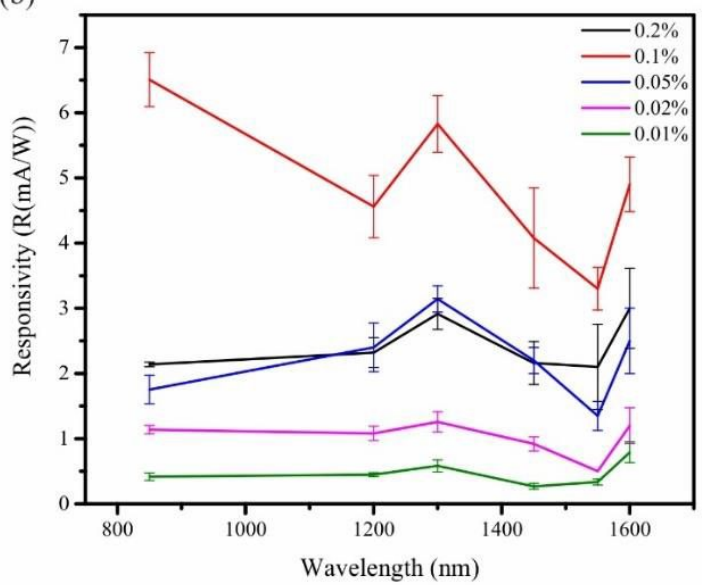

(c)

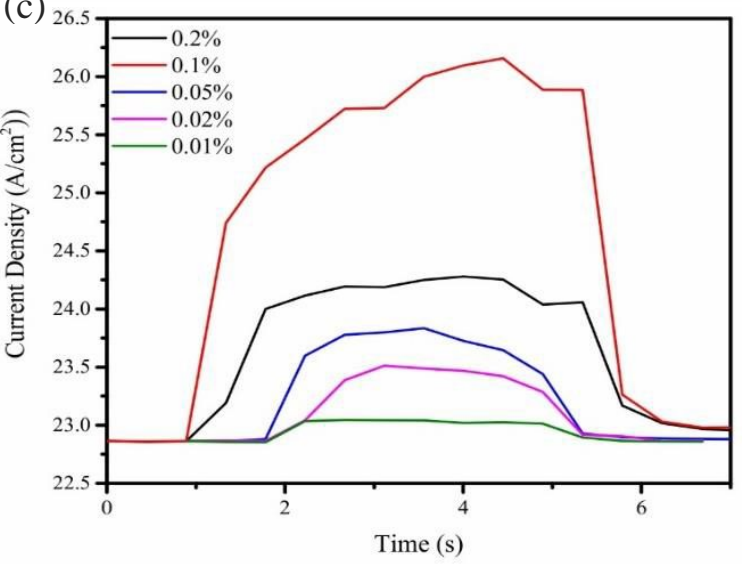

Figure 3. Spectral response of the CNT photo-resistors in visible (a) and infrared (b) region; (c) photocurrent responses of 5 different photo-resistors to $850 \mathrm{~nm}$ IR source. For all cases $1 \mathrm{~V}$ bias is applied on devices.

The above photoresponses implies that $0.1 \%$ CNT contained photo-resistor would be the best choice, due to the highest responsivity among others, used as a low-consumption device that able to detect irradiation in both visible and near infrared region. For that reason, photoresponse of the $0.1 \%$ CNT contained photo-resistor investigated in detail. Figure 4 depict the photoresponse of the corresponding photo-resistor under pulsed illumination with different wavelengths in both visible and infrared regions. Significantly, at an applied bias voltage of $1.0 \mathrm{~V}$, the current increased $7.69 \mu \mathrm{A}$ and further to 158 $\mu \mathrm{A}$ with the illumination by $525 \mathrm{~nm}$ and $405 \mathrm{~nm}$ light sources, respectively. 


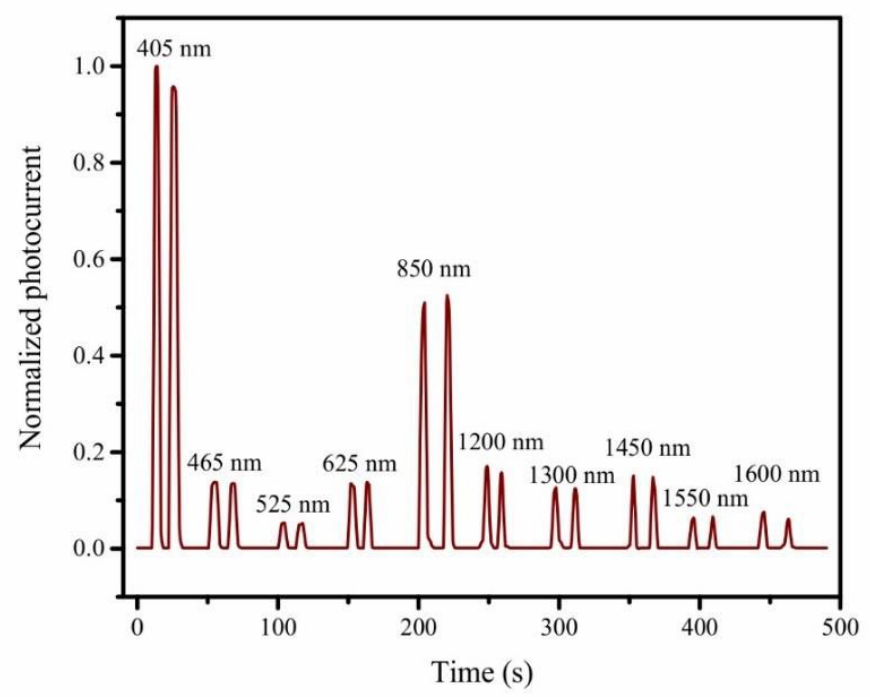

Figure 4. Photoresponse of the $0.1 \%$ CNT contained photo-resistor under visible and infrared illumination at $1 \mathrm{~V}$ bias.

\section{SUMMARY}

In summary, low-consumption broadband photosensitive device was fabricated by using electrospinning method. PVDF nanofibers were acted as a substrate for CNT and PMMA was pedestal for the device. Acid treated CNT showed unexpected compatibility and form a highly conductive network with PVDF nanofibers that can be manipulated by irradiation. We further, investigate the effect of the concentration of CNT on photocurrent. Even base resistivity so as current increases with the amount of CNT drop casted to photo-resistor, largest responsivity was observed in the one contained $0.1 \%$ CNT. In addition, studies showed that, obtained photo-resistors have broadband photoresponse from 400 $\mathrm{nm}$ to $1600 \mathrm{~nm}$ which makes it a promising competitor in telecommunication technologies. Importantly, further investigations are required to fabricate more efficient devices.

\section{ACKNOWLEDGEMENT}

Scanning Electron Microscopy (SEM) images were obtained from Quanta 250 FEG (FEI Corporation, OR, USA) that located in Center for Materials Research in Iztech. We acknowledge the partial support of TUBITAK-2150542 grant. 


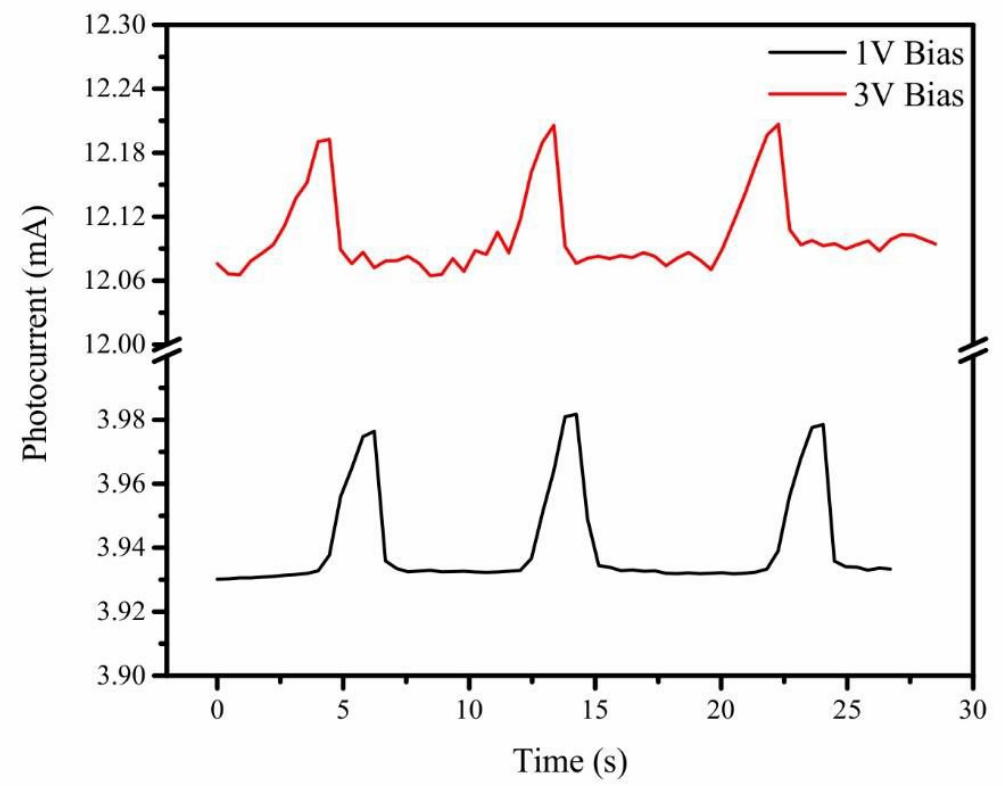

$\mathrm{S} 1$. Photoresponse of the $0.2 \% \mathrm{CNT}$ contained photo-resistor to $850 \mathrm{~nm}$ light source at $1 \mathrm{~V}$ and $3 \mathrm{~V}$ bias.

\section{REFERENCES}

[1] Kim, D.H., et al., "Stretchable and foldable silicon integrated circuits. Science", 2008. 320(5875): p. 507-11.

[2] Kim, D.H., et al., "Flexible and stretchable electronics for biointegrated devices". Annu Rev Biomed Eng, 2012.

14: p. 113-28.

[3] Kim, D.-H., et al., "Inorganic semiconductor nanomaterials for flexible and stretchable bio-integrated electronics. NPG Asia Materials", 2012. 4(4)

[4] Kim, J., et al., "Next-generation flexible neural and cardiac electrode arrays." Biomedical Engineering Letters, 2014. 4(2): p. 95-108.

[5] Lim, S., et al., "Transparent and Stretchable Interactive Human Machine Interface Based on Patterned Graphene Heterostructures." Advanced Functional Materials, 2015. 25(3): p. 375-383.

[6] Kim, D.H., et al., "Stretchable, curvilinear electronics based on inorganic materials." Adv Mater, 2010. 22(19): p. 2108-24.

[7] Kim, D.-H., et al., "Materials for stretchable electronics in bioinspired and biointegrated devices." MRS Bulletin, 2012. 37(03): p. 226-235.

[8] Kim, J., et al., "Stretchable silicon nanoribbon electronics for skin prosthesis." Nat Commun, 2014. 5: p. 5747.

[9] Viventi, J., et al., "Flexible, foldable, actively multiplexed, high-density electrode array for mapping brain activity in vivo." Nat Neurosci, 2011. 14(12): p. 1599-605.

[10] Ying, M., et al., "Silicon nanomembranes for fingertip electronics. Nanotechnology," 2012. 23(34): p. 344004

[11] Kim, D.-H., et al., "Complementary metal oxide silicon integrated circuits incorporating monolithically integrated stretchable wavy interconnects." Applied Physics Letters, 2008. 93(4).

[12] Hata, K., et al., "Water-assisted highly efficient synthesis of impurity-free single-walled carbon nanotubes." Science, 2004. 306(5700): p. 1362-4.

[13] Roh, E., et al., "Stretchable, Transparent, Ultrasensitive, and Patchable Strain Sensor for Human-Machine Interfaces Comprising a Nanohybrid of Carbon Nanotubes and Conductive Elastomers." ACS Nano, 2015. 9(6): p. 6252-61.

[14] Yamada, T., et al., "A stretchable carbon nanotube strain sensor for human-motion detection." Nat Nanotechnol, 2011. 6(5): p. 296-301

[15] Yang, X., et al., "Liquid-mediated dense integration of graphene materials for compact capacitive energy storage." Science, 2013. 341(6145): p. 534-7.

[16] Yücel, M., "FABRICATION OF THIN LAYER POLYMERBASED BIOINTERPHASE FOR BIOSENSING APPLICATION." in Chemistry. June 2016, Izmir Institüte of Technology. 